\title{
Projected economic evaluation of the national implementation of a hypothetical HIV vaccination program among adolescents in South Africa, 2012
}

Nishila Moodley ${ }^{1,2,3^{*}}$, Glenda Gray ${ }^{4,5}$ and Melanie Bertram ${ }^{6}$

\begin{abstract}
Background: Adolescents in South Africa are at high risk of acquiring HIV. The HIV vaccination of adolescents could reduce HIV incidence and mortality. The potential impact and cost-effectiveness of a national school-based HIV vaccination program among adolescents was determined.

Method: The national HIV disease and cost burden was compared with (intervention) and without HIV vaccination (comparator) given to school-going adolescents using a semi-Markov model. Life table analysis was conducted to determine the impact of the intervention on life expectancy. Model inputs included measures of disease and cost burden and hypothetical assumptions of vaccine characteristics. The base-case HIV vaccine modelled cost at US\$ 12 per dose; vaccine efficacy of $50 \%$; duration of protection of 10 years achieved at a coverage rate of $60 \%$ and required annual boosters. Incremental cost-effectiveness ratios (ICER) were calculated using life years gained (LYG) serving as the outcome measure. Sensitivity analyses were conducted on the vaccine characteristics to assess parameter uncertainty.

Results: The HIV vaccination model yielded an ICER of US\$ 5 per LYG (95 \% CI ZAR 2.77-11.61) compared with the comparator, which is considerably less than the national willingness-to-pay threshold of cost-effectiveness. This translated to an $11 \%$ increase in per capita costs from US\$ 80 to US\$ 89. National implementation of this intervention could potentially result in an estimated cumulative gain of 23.6 million years of life ( $95 \% \mathrm{Cl} 8.48-34.3$ million years) among adolescents age 10-19 years that were vaccinated. The 10 year absolute risk reduction projected by vaccine implementation was $0.42 \%$ for HIV incidence and $0.41 \%$ for HIV mortality, with an increase in life expectancy noted across all age groups. The ICER was sensitive to the vaccine efficacy, coverage and vaccine pricing in the sensitivity analysis.

Conclusions: A national HIV vaccination program would be cost-effective and would avert new HIV infections and decrease the mortality and morbidity associated with HIV disease. Decision makers would have to discern how these findings, derived from local data and reflective of the South African epidemic, can be integrated into the national long term health planning should a HIV vaccine become available.
\end{abstract}

Keywords: HIV, Vaccine, Cost-effective, LYG, ICER, Antiretroviral therapy (ART)

\footnotetext{
* Correspondence: nishila.moodley@gmail.com

${ }^{1}$ Perinatal HIV Research Unit, Faculty of Health Sciences University of the

Witwatersrand, PO Box 114 Diepkloof 1864 Johannesburg, South Africa

${ }^{2}$ South African HVTN AIDS Vaccine Early Stage Investigator Program (SHAPe),

Seattle, WA, United States

Full list of author information is available at the end of the article
} 


\section{Background}

South Africa has the largest human immunodeficiency virus (HIV) epidemic in the world [1]. In 2012, 6.4 million South Africans were living with HIV; 203,000 individuals had lost their lives to it and another 395,000 South Africans had acquired the infection [2,3]. South Africa's life expectancy was understandably adversely affected by the considerable burden of HIV disease [4]. However, life expectancy had since increased from 53 years in 2006 to 61 years in 2012, and ensuring its continued improvement remains a priority of the national department of health [5]. The gains made in improving life expectancy are in no small part attributable to 'the largest antiretroviral (ART) rollout in the world' that South Africa has managed to achieve [6]. To sustain this achievement is no mean feat. The growing number of patients previously initiated on ART need to be retained in care. While the public sector retention rate approximates $75 \%$ after one year on treatment, South Africa needs to continuously enroll in excess of 500000 new patients onto ART annually to maintain an ART enrolment ratio exceeding 1.3 [4]. This brings into question the long term sustainability of the ART program considering the massive financial and human resource implications the expansion of ART program entails [7].

Data suggests that close to $25 \%$ of all new HIV infections occurred among young women aged 15-24 years, emphasizing this group as a major driver of the epidemic [2]. The HIV prevalence in this age group is important as it serves as a proxy for HIV incidence. HIV prevalence declined by $18 \%$ in this age group from 2008 to 2012, from $8.7 \%$ to $7.1 \%$, however there remains a need for intensified prevention efforts [8]. Despite massive accomplishments made in establishing the ART program, the women aged $15-24$ years persist as the group with the poorest access to this life-saving treatment. The barriers that young people face in accessing public health services has been well documented [9]. Issues concerning lack of confidentiality and privacy, unfriendly and judgmental attitudes of health care staff and inaccessible clinic hours persist $[10,11]$. It was against this backdrop that the re-engineering of primary health care in South Africa targeted the development of a school-based sexual and reproductive health service as a priority [12].

The current HIV prevention program has enjoyed limited success in tackling the high rate of new infections in South Africa, highlighting the need for an alternative intervention. Vaccines are regarded at the most costeffective prevention intervention in the world [13]. RerksNgam et al tested the first HIV vaccine regimen (RV144/ Thai Trial) to show moderate vaccine efficacy in humans in Thailand (2009) [14]. The study evaluated a primeboost strategy, priming with a recombinant canarypox vector (ALVAC-HIV[vCP1521]) administered at baseline, then at week 4, 12 and 24 with recombinant glycoprotein 120 subunit vaccine (AIDSVAX B/E) boosts given with the ALVAC at weeks 12 and 24.

The prime-boost HIV vaccine regimen used resulted in modest efficacy of $31 \%$ over 3.5 years [14]. While the effects were not durable, they were indeed promising. After undergoing modifications to optimize the HIV vaccine regimen by making it Clade $\mathrm{C}$ specific and changing the protein and adjuvant, a potential vaccine regimen was entered into Phase I/IIb clinical trials at six major South African centers to assess safety and immunogenicity (HIV Vaccine Trial Network (HVTN) 100 study) [15]. Additionally, a pivotal phase IIb/III HIV vaccine efficacy trial is planned to take place in South Africa designated HVTN 702, which will evaluate the same regimen [as HVTN 100], should HVTN 100 prove to be immunogenic.

The aim of this analysis was to guide decision makers in assessing the value of national implementation of a potential HIV vaccine among school-based adolescents in South Africa. The work determined the impact of vaccination on HIV disease burden and associated health costs, and evaluated the cost-effectiveness and potential changes in life expectancy based on the premise that school-based care would address the issues of equity and accessibility in health care that adolescent South Africa faces.

\section{Methods}

The study methodology was compliant with the reporting guidelines of the Consolidated Health Economic Evaluation Reporting Standards (CHEERS) statement [16].

\section{Study overview}

Ten year old adolescents attending South African schools in 2012 were considered for vaccination. This intervention program was introduced as part of the national health initiative to introduce school-based sexual and reproductive health services [12], and targeted learners prior to the onset of sexual activity. The cohort was modelled through a lifetime horizon of 70 years inclusive, which exceeded the current estimated life expectancy of 60.6 years in South Africa [3]. The rationale for this was that life expectancy is rapidly changing in the South African environment and this cohort was considered to probably have a greater life expectancy. The assumption made was that the HIV vaccine would be incorporated into the South African Expanded Program of Immunization and would be administered at school level. The health service provider (provider) perspective was adopted as the information generated was intended to inform national health decision making. The hypothetical HIV vaccine was modelled as a prevention strategy that reduced the HIV disease burden and associated mortality. The vaccine strategies were considered against the system of HIV counselling and testing (HCT) and the national rollout of ART that constituted 
the standard of care (comparator model) in South Africa $[17,18]$. The intervention model combined the current standard of care with the HIV vaccination strategy as both programs would be delivered simultaneously. A discount rate of $3 \%$ was applied to the economic costs and health outcomes, as recommended by the World Health Organization CHOosing Interventions that are Cost-Effective (WHO-CHOICE) guidelines [19]. The epidemiology of South African epidemic is described in Table 1.

\section{Outcome measures}

Life years gained (LYG) was measured in terms of its impact on mortality. The LYG concept represents a modified mortality measure which considers remaining life expectancy. More weight is accrued to the life of a young child than an elderly person, because saving the life of a young child will accrue more life years than saving the life of an elderly person. The life years are calculated as the "remaining life expectancy at the point of each averted death" [20]. Life tables are generally setting specific or standardized for a geographic area. Using the information generated in these life tables, we are able to derive life expectancies for a specific population.

The HIV vaccine described for implementation was hypothetical as it is currently undergoing Phase I/II clinical trials. The HIV vaccine characteristics were determined by the target product profile formulated by the Pox-Protein Private Public partnership (P5), developed to build on the success of the RV144/Thai trial and evaluate potential HIV vaccine candidates to determine their public health impact [21]. The regimen included in this economic evaluation mirrored the ongoing HVN 100 study which adapted the ALVAC prime ALVAC/gp120/adjuvant boost of the RV144/Thai trial but added an additional ALVAC/ gp120/adjuvant boost at month 12 . This boost at month 12 was added to circumvent the waning of the immune response documented in the RV144/Thai trial a year after initial vaccine administration.
The estimated vaccination coverage was $60 \%$ (range: 40 $70 \%$ ). This represents a slight underestimation of the $68 \%$ reported for coverage of the 3rd dose of diphtheria, tetanus and pertussis toxoid (DTP3) which has been validated as a proxy for national immunization performance [22]. The base-case HIV vaccine modelled cost US\$ 12 per dose (range: US\$ 2-24), had a vaccine efficacy of $50 \%$ (range: 30-70\%) and the duration of protection of 10 years (achieved through the administration of annual boosters). The declining immunity reported in the RV144/Thai trial (particularly in the year following administration) reaffirmed the need for booster injections. Annual boosters may be far from pragmatic but merely represented an overestimation of costs in this evaluation. The vaccine price of US\$ 12 was roughly based on the human papillomavirus (HPV) vaccine available on government tender at US\$ 17 . Markedly reduced vaccines prices deemed plausible given the strides made in negotiating lower priced ART medications and HPV vaccines in the public sector [23, 24]. Pooled utilities relating to HIV/AIDS (acquired immunodeficiency syndromes) were derived from a meta-analysis and were used for the cost-effectiveness analyses of HIV related interventions [25].

\section{Study inputs}

Input parameters are shown in Table 2. Estimated vaccination coverage of $60 \%$ of adolescents approximated 6 million individuals receiving the initial course. Delivery of health services was conducted at the schools. HIV related costs were estimated identified from the 2013 national HIV treatment guideline [18]. Patients would be consulted by primary health care (PHC) nurses and more complicated cases would be referred. Pharmaceutical costs included ART, treatment of sexually transmitted infections (STI) and condoms. In addition to the costs accumulated in the comparator group, the intervention included the vaccine and its delivery. Laboratory tests conducted by the National Health Services Laboratory, costing of medication, consumables and additional pharmaceuticals and

Table 1 South African population by age groups exploring ARV treatment access. The HIV epidemiology of South Africa is described. The treatment shortfall represents those eligible for ART but unable to access it

\begin{tabular}{llllll}
\hline Age groups & Population & Susceptible & Prevalence $^{\mathrm{a}}$ & On ARV treatment & Treatment shortfall $^{\mathrm{b}}$ \\
\hline $10-19$ & 10264690 & 9982612 & 282078 & 78176 & 163605 \\
$20-29$ & 11010305 & 9386287 & 1624018 & 411831 & 941930 \\
$30-39$ & 9008794 & 6521402 & 2487392 & 775604 & 1442687 \\
$40-49$ & 4479445 & 3329718 & 1149727 & 358501 & 666842 \\
$50-59$ & 3367397 & 2883570 & 483827 & 204740 & 280620 \\
$60+$ & 3665571 & 3534983 & 130588 & 55260 & 75741 \\
Totals & 41796202 & 35638572 & 6157630 & 1884112 & 3571425 \\
\hline
\end{tabular}

${ }^{a}$ Shisana O et al. South African National HIV Prevalence, Incidence and Behaviour Survey, 2012. Cape Town, HSRC Press. 2014

bUN Joint Program on HIV/AIDS (UNAIDS). The Gap Report. 2014 
Table 2 Parameter costs and economic considerations. The estimates were obtained from relevant South African literature for the year 2012

\begin{tabular}{|c|c|c|c|}
\hline HIV vaccine characteristics & Value & (Range) & Reference \\
\hline Coverage & $60 \%$ & $(40-70)$ & Assumption \\
\hline Price (US\$) & 12 & $(2-24)$ & [47] \\
\hline HIV vaccine efficacy & $50 \%$ & $(30-70)$ & Assumption \\
\hline Economics & Value & (Range) & Reference \\
\hline Cost discount rate & $3.0 \%$ & $(0-6 \%)$ & [19] \\
\hline Outcome discount rate & $3.0 \%$ & $(0-6 \%)$ & [19] \\
\hline International comparison (ZAR: 1US\$) & ZAR 8.21 & - & [26] \\
\hline HIV disease related costs & Distribution & Value & Reference \\
\hline \multicolumn{4}{|l|}{ HIV prevention programme } \\
\hline HIV vaccine & - & 12 & [21] \\
\hline Vaccine delivery per dose & Gamma & 17 & {$[48-52]$} \\
\hline Existing prevention programme (incl. HR) & Gamma & 65 & [49-53] \\
\hline Voluntary counselling and testing (VCT) (per test) & Gamma & 23 & {$[49,50]$} \\
\hline Cost of HIV rapid testing & Gamma & 2 & {$[49,50]$} \\
\hline \multicolumn{4}{|l|}{ Current HIV programme (annual costs) } \\
\hline Asymptomatic treatment (not on ART) & Gamma & 131 & {$[18,53]$} \\
\hline Symptomatic treatment (not on ART) & Gamma & 137 & {$[18,53]$} \\
\hline AIDS treatment (not on ART) & Gamma & 182 & {$[18,53]$} \\
\hline Patient on ART (average) & Gamma & 424 & {$[18,23]$} \\
\hline \multicolumn{4}{|l|}{ ART cost (annual) } \\
\hline First-line regimen & Gamma & 10 & [23] \\
\hline Second-line regimen & Gamma & 27 & [23] \\
\hline Third-line regimen & Gamma & 173 & [23] \\
\hline \multicolumn{4}{|l|}{ Laboratory costs (annual) } \\
\hline First-line regimen (first year) & Gamma & 17 & {$[18,53]$} \\
\hline First-line regimen (subsequent years) & Gamma & 46 & {$[18,53]$} \\
\hline Second-line regimen & Gamma & 46 & {$[18,53]$} \\
\hline Third-line regimen & Gamma & 92 & {$[18,53]$} \\
\hline Not on ART & Gamma & 65 & [53] \\
\hline
\end{tabular}

valuations of medical personnel cost based on the Uniform Patient Fee Schedule (UPFS) were sourced from the National Department of Health. All costs were adjusted to the common year 2012. Costs were converted from South Africa rand (ZAR) to United States dollar (US\$) using the average exchange rate for 2012, thus allowing for international comparison (US\$ $1=$ ZAR 8.21) [26]. HIV related disease transition probabilities were obtained from the South African literature and are shown in Table 3.

\section{Model based economic evaluation} Semi-Markov model development

Data capture and analysis was conducted in Microsoft Excel $^{\bullet}$ (Version 2010) (Microsoft Corp., Redmond, WA). Ersatz version 1.2 (www.epigear.com), a boot-strap add-
Table 3 Disease transition probabilities showing annual progression risk. The possibility of transition from one HIV health state to the next is described. The estimates were obtained from relevant South African literature for the year 2012

\begin{tabular}{llll}
\hline Parameter & Distribution & Estimate & Reference \\
\hline Change in HIV disease state & & & \\
Asymptomatic to symptomatic & Beta & 0.32 & {$[54]$} \\
Symptomatic to AIDS & Beta & 0.20 & {$[55]$} \\
AIDS to death & Beta & 0.21 & {$[54]$} \\
Change in drug regimens & & & \\
First-line to second-line & Beta & 0.10 & {$[55]$} \\
Second-line to third-line & Beta & 0.01 & {$[56]$} \\
\hline
\end{tabular}


in application for Excel, was used to perform the uncertainty analysis.

The simulation ran a semi-Markov simulation with annual cycles (Fig. 1). Tunnel states could be added to the semi-Markov model that countered the 'memoryless' nature inherent in the models. The vaccine was offered on a voluntary basis to adolescents from the age of ten years. The model comprised eight health states. All individuals were considered HIV negative and healthy at the start of the model (State 1). The coverage rate determined who moved into a vaccinated (State 2 ) or unvaccinated (State 3) state. All individuals may transition into an asymptomatic HIV state (State 4). Individuals who seroconverted to HIV positive were started on ART when eligible. Asymptomatic individuals may progress to a symptomatic (State 5) or AIDS (State 6) state. Every HIV infected individual may enter the treatment pool (State 7) which was sub-classified as 1st, 2nd and 3rd line ART regimens. Every aforementioned health state may transition to death (State 8). Each cycle carries a probability of remaining in the current health state or transitioning to another with the arrows representing the transition probabilities from one state to another. Once the vaccine had been stopped, event rates were assumed to be the same for both arms of the study.

One-way sensitivity analyses evaluated the impact of single assumptions on cost and outcomes. Probabilistic sensitivity analysis (PSA) with a bootstrapping technique of 1000 iterations was used to explore the uncertainty in the model and evaluate the robustness of the results. These results were presented as cost-effectiveness scatter plots and cost-effectiveness acceptability curves. The
PSA data generated was used to determine if the intervention fell below the willingness-to-pay (WTP) threshold. As South Africa does not have a pre-defined WTP threshold, the Gross Domestic Product (GDP) per capita (2012) was used as a proxy in accordance with the WHO Guide to Cost-Effective Analysis [19, 27]. The WTP threshold was thus defined as US\$ 7508 (ZAR 61 641) per quality adjusted life year (QALY) gained. The GDP per capita range was adapted from the 'value of statistical life' literature and is theoretically the value of an additional healthy life year [28]. It is used in the context of this study against LYG (rather than the convention QALY) as there is no other alternative available to indicate cost-effectiveness in South Africa.

\section{Cost and cost-effectiveness of a national HIV vaccination program}

The programmatic costs and health implications of a vaccine implemented at US\$ 12 per dose and $60 \%$ coverage was determined and this was considered the base case. Using PSA techniques, we were able to estimate the change in cost per capita, the approximate cost per LYG and finally the cost per death averted at different vaccines prices per dose. The change from the base cost for the program cost was compared with the baseline vaccine implementation at US\$ 12 per dose.

\section{Life table analysis}

A multi-state life table approach was used to describe the differential morbidity and mortality of a population under two alternative interventions [29]. The alternatives were

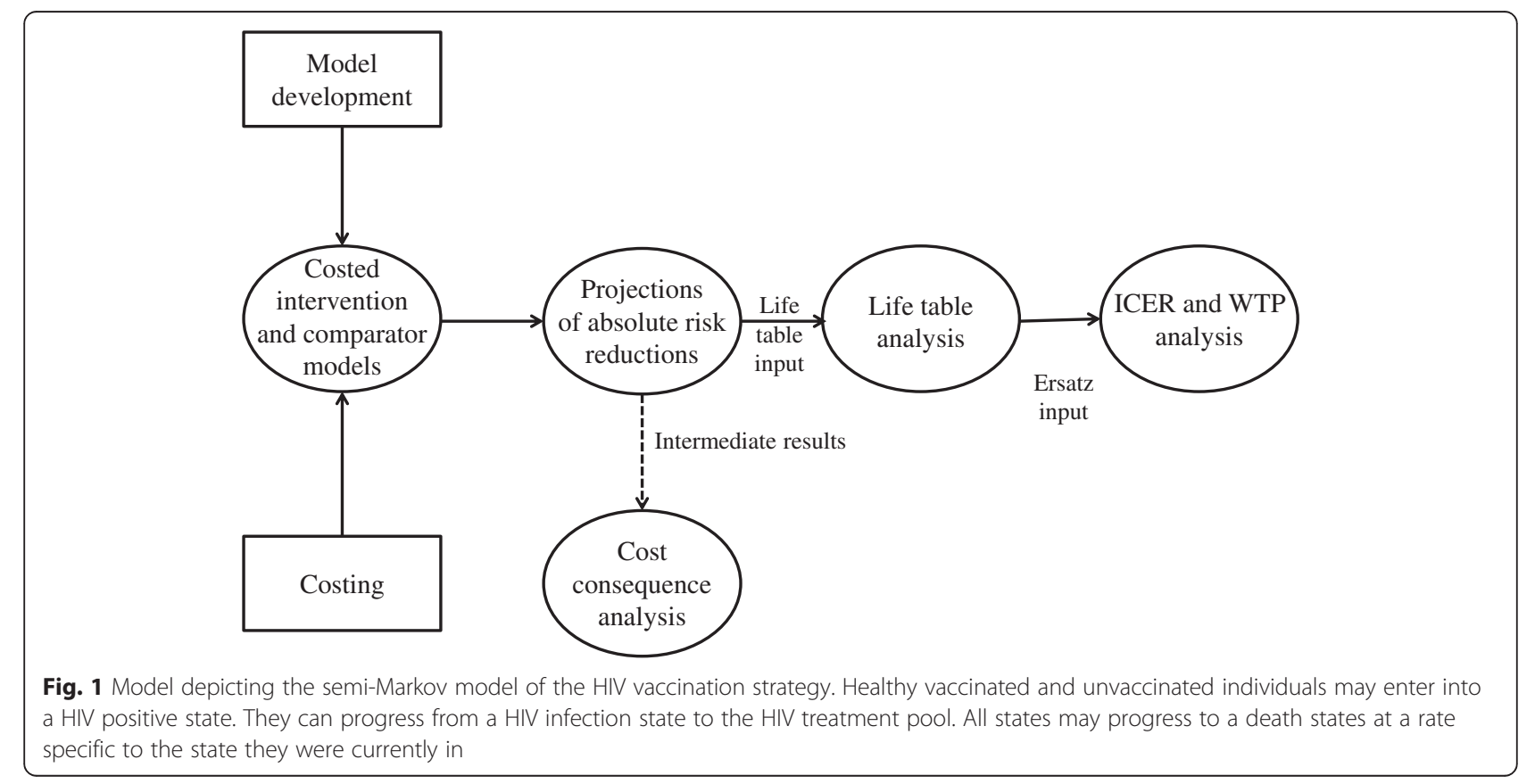


the reference population displaying the HIV associated mortality experienced by the South African adolescent population under the comparator model compared with the outcomes from the adolescent population when exposed to the intervention (which was the vaccine strategy in addition to the comparator model). Disease related mortality was referenced from the literature (Table 3). The study used a cohort life table methodology which calculated the probability of death of a generation (cohort) over the course of their lifetime. Cohort life tables use agespecific mortality rates related to specific cohorts which allow for known and projected changes in mortality [30]. Within a standard life table, the disease related mortality was separated from national mortality (as shown in Eq. 1):

$$
M_{\text {tot }}=M_{\text {dis }}+M_{\text {other }}
$$

Where $M_{\text {tot }}$ is the total mortality identified in the age/ sex group, $M_{\text {dis }}$ is the mortality attributed to the disease state and $M_{\text {other }}$ is the mortality attributed to all other causes.

The prevalence estimates for HIV was obtained from South African National HIV Prevalence, Incidence and Behaviour Survey, 2012 [2]. The ratio between the comparator and the intervention groups was used to calculate the relative reduction in HIV related mortality attributable to the intervention (reflected in Eq. 2). This reduction was applied in the life table allowing for comparisons to be made including the life expectancy, individuals surviving and the cumulative years lived.

$$
R R_{m}=\frac{M_{i}}{M_{c}}
$$

Where $R R_{m}$ is the mortality risk reduction, $M_{i}$ is the mortality risk in the intervention group and $M_{c}$ is the mortality risk in the comparator group.

Values were entered into a life table to estimate the impact of the intervention on life expectancy and the number of life years gained. Generally, a life table estimates the mortality experience of a population and calculates the life expectancy from birth [31]. The life expectancy calculated from a life table is represented by the following formula (Eq. 3) [32]:

$$
e_{x}=\frac{T_{x}}{I_{x}}
$$

Where $e_{x}$ is the life expectancy at age $\mathrm{X}, T_{x}$ is the cumulative person years lived after age $\mathrm{X}$ and $l_{x}$ are the individuals alive at beginning of age $\mathrm{X}$.

The difference in cumulative years lived between the intervention and comparator groups were used in the incremental cost-effectiveness ratios (ICER) calculations. The ICER represents the difference in costs between strategies and the difference in effects (e.g. LYG) between strategies (Eq. 4). The unit of measurement of the ICER is US\$ per LYG gained.

$$
I C E R=\frac{C_{2}-C_{1}}{E_{2}-E_{1}}=\frac{\Delta C}{\Delta E}
$$

Where $C_{1}$ and $E_{1}$ are the costs and effects of the standard of care (comparator), and $\mathrm{C}_{2}$ and $\mathrm{E}_{2}$ are the costs and effects of the intervention.

\section{Years of potential life lost}

The years of potential life lost (YPLL) is used to measure the incidence of 'premature' mortality that occurs within a population to an age at which the death is considered untimely [33, 34]. The YPLL concept quantifies social and economic loss as a result of premature death, and has been useful in assessing specific causes of death targeting younger age groups [35]. The principle of YPLL incorporates the age at death, and the calculation is able to mathematically weight the total deaths by applying values to death at each age (Eq. 5) [34-36].

$$
Y P L L=\Sigma\left({ }_{n} \mathrm{~d}_{x}^{i}\right) \times[70-(n \times 5)]
$$

Where ${ }_{n} \mathrm{~d}_{x}^{i}$ is the number of deaths due to HIV/AIDS from age $x$ to age $x+n$ and $n$ is the width of the age interval (in this study ten-year age intervals were used) and 5 represents the number of years till the midpoint of the age interval is reached.

\section{Cost consequence analysis}

The absolute risk reduction (ARR) was then measured as a percentage. This represented the change in the risk of an outcome of the intervention in comparison to the comparator. It was calculated as the difference in the mean values of the parameter of interest and an example of the calculation is shown in Eq. 6.

HIV incidence comparator $_{\text {- HIV incidence }}$ intervention $=\mathrm{ARR}[\%]$

Where the HIV incidence comparator and HIV incidence intervention represented mean percentages and the difference in values was the absolute risk reduction percentage.

The difference in per capita costs with and without the intervention was then divided by the ARR values obtained for HIV incidence and HIV mortality to yield the cost per percentage reduction in disease. The outcomes for both the ARR and the per percentage reduction in disease burden was described by gender to highlight the areas of greatest impact. 


\section{Model assumptions}

All participants entering the model were considered sexually naïve. Drop-out rates were not accounted for as all children of school-going age were assumed to be attending school. The model assumed that the rollout and uptake of HIV counselling and testing (HCT) strategies and the national rollout of the HIV vaccination strategy occurred within the school-based health services that provided comprehensive care to all socio-economic levels of learners. Finally, the model assumed good uptake of school-based health services given the provision of care in a familiar and safe environment with no encroachment on school attendance. As no formal pilot studies have been reported, there remains no validation of this assumption.

\section{Ethical consideration}

Ethical approval for the study was obtained from the Human Research Ethics Committee (Medical) of the University of the Witwatersrand.

\section{Results}

\section{Costs of models}

The annual per capita cost of the comparator was US\$ 80. Annual HIV vaccination per capita cost was calculated at US\$ 89, representing an $11 \%$ increase in costs. Table 4 describes the complete breakdown of these costs. There is no appreciable difference in human resources and laboratory costs associated with the vaccine intervention, though the intervention does represent a saving on both these costs. However, the intervention does predict an increase (31\%) in pharmaceutical costs driven by the need for vaccine boosters to attain durable protection. The vaccine price considered in Table 4 was US\$ 12.

\section{Uncertainty analysis \\ The cost and cost-effectiveness of a national HIV vaccination program}

Implementing a South African national HIV vaccination program at the base vaccine cost of US $\$ 12$ per dose (Table 5) would be considered cost-effective at US\$ 5 per LYG. When benchmarking this against the WHO cost-effectiveness criteria (US\$ 7508 per QALY gained), a HIV vaccine at US\$ 12 is deemed highly cost-effective. However, introduction of the HIV vaccine at considerably reduced price per dose will significantly impact the future sustainability of the program. At the low vaccine cost of US\$ 6 , the program cost will be reduced by $5 \%$ (US\$ 52 million) of the base vaccination program; and will result in an ICER of US\$ 2 per LYG. The very low vaccine price of US\$ 2 would yield even better results an ICER of US\$ 1 per LYG with a $9 \%$ reduction (US\$
84 million) in the program costs compared with the baseline vaccination strategy.

\section{Impact of coverage on cost and life expectancy}

Table 6 explores the impact of initial vaccine and subsequent booster coverage on cost and life expectancy. To vary the coverage annually would be computationally challenging, hence the combination of the initial vaccine and the administration of the annual booster was considered as a continuum i.e. if the initial vaccine coverage was $40 \%$, then the booster coverage considered was also $40 \%$. Increasing the vaccine coverage would result in significantly increased financial investment. However increasing coverage also translated to improved life expectancy. The increased cost has to be weighed against the improved health outcomes, before the strategy is deemed cost-effective. There would also have to be consideration of the impact of other vaccine characteristics.

\section{Probabilistic sensitivity analysis ICER and WTP results}

The uncertainty around the ICER was assessed using probabilistic sensitivity analysis. The HIV vaccine intervention yielded an ICER of US\$ 4.98 per LYG (95\% CI ZAR 2.77-11.61). National projections of the intervention programme were estimated to cost US\$ 1017 million annually. This represents a US\$ 104 million (11\%) increase on the comparator cost of US\$ 913 million. Aside from the need for boosters driving the cost, it should be borne in mind that the vaccine is anticipated to reach approximately 6 million HIV negative 10-19 year old adolescents compared with the comparator strategy providing ART to 78126 adolescents of the same age group. The intervention, however, would translate to a mean cumulative gain of 23.6 million LYG (95\% CI 8.48-34.3 million years) in the population. Apart from demonstrating the cost-effectiveness of the vaccine intervention, Fig. 2 was designed to evaluate the impact of differing vaccine efficacies on the ICER. At a vaccine efficacy of $30 \%$, the iterations lie on either side of the WTP threshold indicating that the intervention may not be cost-effective. However, at the vaccine efficacy of $50 \%$ and $70 \%$, most iterations were considerably below the GDP per capita of South Africa, 2012. Based on this GDP, the intervention would be considered below the WTP threshold defined by the World Health Organization (WHO) and thus deemed to be highly cost-effective [19].

\section{Life expectancy and potential years of life lost}

The simulation of the life table results are presented in Table 7. Application of the intervention in the 10-19 year age group resulted in a 2.5 year increase in life expectancy, as well as a significant increase in cumulative 
Table 4 Model components and cost comparison of the HIV vaccination program (US\$). Complete breakdown of costs relating to the intervention and the comparator. The intervention comprises both the vaccine strategy and the comparator costs

\begin{tabular}{|c|c|c|c|c|}
\hline \multirow[t]{2}{*}{ Cost category } & \multicolumn{4}{|c|}{ Per capita expenditure } \\
\hline & Intervention & Comparator & Difference & (\% change) \\
\hline Laboratory & 12.73 & 13.09 & 0.35 & $(-2.78)$ \\
\hline HIV rapid testing & 1.06 & 1.41 & & \\
\hline CD4 count & 4.05 & 4.05 & & \\
\hline Pap smear & 1.13 & 1.13 & & \\
\hline Viral load & 5.95 & 5.95 & & \\
\hline Creatinine & 0.53 & 0.53 & & \\
\hline Pharmaceuticals & 39.86 & 30.21 & 9.64 & $(+31.92)$ \\
\hline STI treatment & 1.11 & 0.92 & & \\
\hline Condom distribution & 1.35 & 1.12 & & \\
\hline Contraception & 0.77 & 0.63 & & \\
\hline Anti-retroviral therapy & 27.34 & 27.34 & & \\
\hline Vaccine & 8.94 & 0.00 & & \\
\hline Vaccine delivery $^{a}$ & 0.15 & 0.00 & & \\
\hline Bactrim ${ }^{\oplus}$ prophylaxis & 0.20 & 0.20 & & \\
\hline Human resources & 35.94 & 36.67 & 0.63 & $(-1.76)$ \\
\hline PHC nurse & 20.70 & 22.24 & & \\
\hline Counsellor & 11.03 & 11.81 & & \\
\hline Enrolled nursing assistant & 1.68 & 0.00 & & \\
\hline Medical officer & 0.46 & 0.46 & & \\
\hline Medical specialist & 2.07 & 2.07 & & \\
\hline Transport ${ }^{b}$ & 0.47 & 0.47 & - & - \\
\hline Total & 89.00 & 80.34 & & \\
\hline
\end{tabular}

Vaccine delivery includes the needle, syringe and alcohol swab for administration

${ }^{b}$ Calculated from average car rental cost incurred in providing a school-based service

gain of years lived in the age group. Importantly, as a result of the increase in life expectancy noted in the 10-19 year group, there was a reported increase documented in the subsequent age groups. The PYLL from HIV/AIDS contributing to 'premature' death is also given in Table 7. It is here that the impact of (the vaccine is demonstrated as there is a years of life lost without the vaccine $(70$ 640) is considerably higher than the years lost with the vaccine intervention (48 400).

\section{Cost consequence results}

The 10 year absolute risk reductions in HIV associated mortality and incidence potentially offered by the HIV

Table 5 Cost -effectiveness of a national HIV vaccination program at varied vaccine prices, 2012. The programmatic cost implications of varying the vaccine cost per dose were examined. The cost values reflect annual expenditure. At baseline (shaded), a vaccine at the cost of US\$12 per dose would result in an annual cost of approximately US\$1017 million. This represents a US\$ 9 increase from the base cost per capita (Table 4). All other values have been calculated relative to the base vaccination strategy

\begin{tabular}{|c|c|c|c|c|c|c|c|}
\hline \multicolumn{2}{|c|}{ Vaccine pricing } & \multicolumn{3}{|c|}{ Program cost (millions) } & \multicolumn{3}{|c|}{ Cost per } \\
\hline \multirow{2}{*}{$\frac{\text { Structure }}{\text { Very low }}$} & \multirow{2}{*}{$\frac{\text { Per dose }}{2}$} & \multirow{2}{*}{$\begin{array}{l}\text { Total } \\
933\end{array}$} & \multicolumn{2}{|c|}{ Change from base (\%) } & \multirow{2}{*}{$\begin{array}{l}\text { Capita }^{a} \\
1\end{array}$} & \multirow{2}{*}{$\frac{\mathrm{LYG}}{1}$} & \multirow{2}{*}{$\frac{\text { Death averted }}{421}$} \\
\hline & & & -84 & $(-9)$ & & & \\
\hline Low & 6 & 967 & -50 & $(-5)$ & 4 & 2 & 1106 \\
\hline Base cost & 12 & 1017 & - & - & 9 & 4 & 2131 \\
\hline Medium & 18 & 1067 & 50 & $(+5)$ & 13 & 6 & 3161 \\
\hline High & 24 & 1118 & 101 & $(+10)$ & 18 & 8 & 4189 \\
\hline
\end{tabular}

\footnotetext{
${ }^{a}$ increase in cost per capita
} 
Table 6 One-way sensitivity analysis of coverage on health outcomes. By varying the coverage rates, we are able to demonstrate how an increased number of doses drive the intervention costs up

\begin{tabular}{llllll}
\hline & \multicolumn{2}{l}{ Cost (million US\$) } & \\
\cline { 2 - 4 } Coverage & Comparator & Intervention & Increase in cost & Life expectancy \\
\hline $40 \%$ & 913 & 982 & 70 & 54.6 years \\
$60 \%$ & 913 & 1017 & 104 & 55.5 years \\
$70 \%$ & 913 & 1034 & 122 & 55.9 years \\
\hline
\end{tabular}

vaccine intervention was projected using data modelled. Table 8 described a detailed breakdown of costs to highlight the differences in vaccine impact between the genders. While all scenarios reflected an improvement in HIV related health outcome, the reduction in HIV incidence among females was notable $(0.53 \%)$, particularly given their high burden of disease.

\section{Discussion}

The study aimed to assess the cost-effectiveness of national rollout of the hypothetical HIV vaccine to schoolbased adolescents. The South African HIV epidemic is widely acknowledged to be generalized, with adolescents and young adults disproportionately at risk for HIV [37]. In 2013, South Africa reported $16 \%$ of the global HIV incidence despite concerted efforts at the national level ranging from increasing ART distribution by $75 \%$ between 2009 and 2011 to boasting the largest and most established condom distribution program in the world $[2,38]$. This earmarked adolescents as a key population to be reached if HIV prevention strategies are to impact incidence and if HIV mortality rates are to be significantly curtailed [37]. While the introduction of a potential HIV vaccine in schools represents a significant financial investment, the health outcomes in terms of improved life expectancy, markedly decreased potential years of life lost and decreases in HIV mortality and incidence are substantive. Life expectancy was equally influenced by vaccine coverage rates, while the assessment of cost-effectiveness was found to be sensitive to the vaccine efficacy.

The life table findings together with the conventionally accepted thresholds for cost-effectiveness being met demonstrate the financial plausibility of HIV vaccine implementation [19]. Importantly, the vaccine remained cost-effective even at higher prices per dose examined but at substantially greater programmatic costs. Annual HIV vaccination represents a substantial increase in costs per capita at base coverage of $60 \%$ of HIV negative adolescents. This constitutes a significant investment considering the intense competition of several competing burdens of disease on a constrained South African health budget [39]. As much as the long term financial sustainability of the burgeoning ART program has been brought into question, the implementation of a HIV vaccine program over several decades may prove equally daunting. It is important to bear in mind that the comparator cost reflects those currently on treatment (excluding the treatment shortfall of approximately $58 \%$ [1]) and thus represents a gross underestimation of what we should be paying if those unable to access treatment were indeed able to access it. Another major consideration is

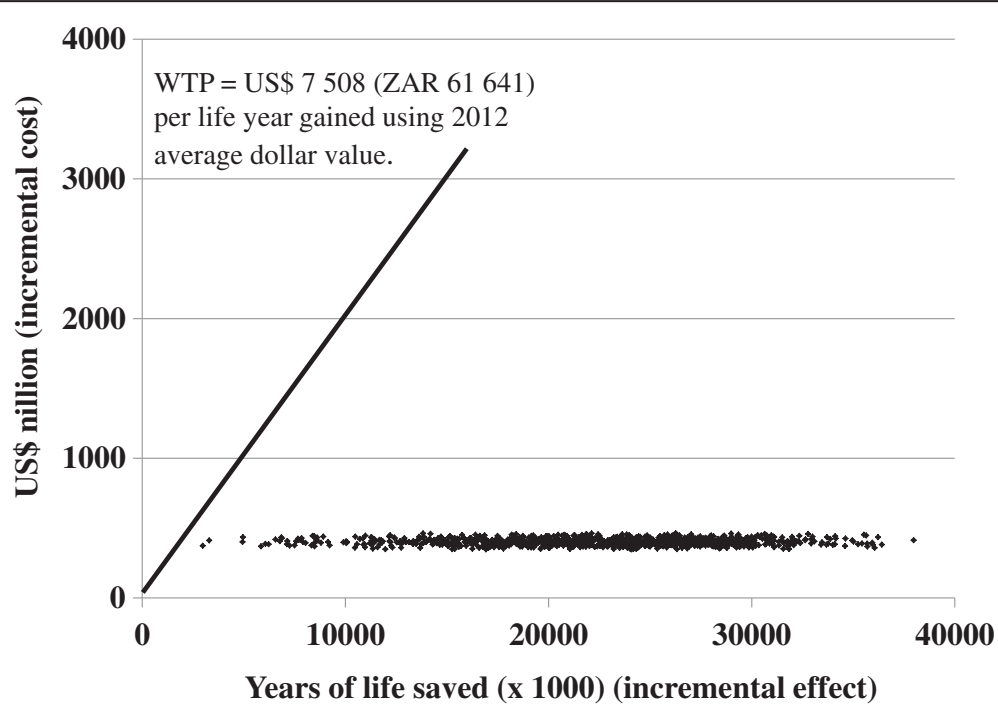

Fig. 2 Willingness-to-pay analysis explored by varying vaccine efficacy. This figure shows the scatter plot of the costs and health outcomes from the probabilistic sensitivity analysis. The incremental cost is the difference in costs between the current treatment program and the vaccine program. Similarly, the incremental effect reflects the difference in health outcomes between the vaccine program and the current treatment program. The health outcomes are measured in years of life saved 
Table 7 Life table analysis and YPLL for 10-19 year age group

\begin{tabular}{|c|c|c|c|c|c|c|}
\hline \multirow{2}{*}{$\begin{array}{l}\text { Age }(x) \\
\text { Life expectancy }\end{array}$} & \multicolumn{3}{|c|}{ No vaccination (comparator) } & \multicolumn{3}{|c|}{ Vaccination (intervention) } \\
\hline & $\begin{array}{l}\mathrm{I}_{\mathrm{x}} \\
\text { (millions) }\end{array}$ & $\begin{array}{l}\mathrm{T}_{\mathrm{x}} \\
\text { (millions) }\end{array}$ & $e_{x}$ & $\begin{array}{l}\mathrm{I}_{\mathrm{x}} \\
\text { (millions) }\end{array}$ & $\begin{array}{l}T_{x} \\
\text { (millions) }\end{array}$ & $e_{x}$ \\
\hline $10-19$ & 10.0 & 529.1 & 53.0 & 10.0 & 553.6 & 55.5 \\
\hline $20-29$ & 9.8 & 429.9 & 43.7 & 9.9 & 454.4 & 46.1 \\
\hline $30-39$ & 9.3 & 334.4 & 36.1 & 9.4 & 358.2 & 38.2 \\
\hline $40-49$ & 8.3 & 246.8 & 29.9 & 8.6 & 268.3 & 31.2 \\
\hline $50-59$ & 7.1 & 170.0 & 23.9 & 7.6 & 187.2 & 24.6 \\
\hline $60+$ & 5.8 & 105.5 & 18.2 & 6.3 & 117.4 & 18.6 \\
\hline \multicolumn{7}{|l|}{ YPLL } \\
\hline $10-19$ & & 70640 & & & 48400 & \\
\hline
\end{tabular}

The movement of the vaccinated population aged $10-19$ years of age is tracked through the life table. Columns $I_{x}$ describes the impact of the intervention in terms of mortality reduction, columns $T_{x}$ reflects the combined years lived with and without the intervention and columns $e_{x}$ reflect the increase in life expectancy attributable to the intervention

$\mathrm{I}_{\mathrm{x}}$ - individuals surviving, $\mathrm{T}_{\mathrm{x}}$ - cumulative years lived, $\mathrm{e}_{\mathrm{x}}$ - remaining life expectancy at age $\mathrm{x}$

that the upscaling of ART may not impact the HIV incidence as definitively as a primary preventative strategy may. It must be remembered that while averting infections has a cost attached from a government perspective, it may also give rise to the substantial financial gains of reducing the demand for ART [40].

South Africa has successfully negotiated reduced pricing for ART and HPV vaccines in the past, and this bodes well for future procurement of HIV vaccines [23, 24], as the price is undetermined at this point. If vaccine development fails to reduce the number of annual boosters required to maintain protection, then the pricing represents a key factor in deciding the costeffectiveness of the intervention. Apart from the economic impact, HIV vaccine implementation has the capacity to influence long term health outcomes. The mean cumulative gain of LYG could support efforts to improve life expectancy in the country, an area

Table 8 Disease risk reduction and cost consequences. The absolute risk reduction was estimated over a 10 year period

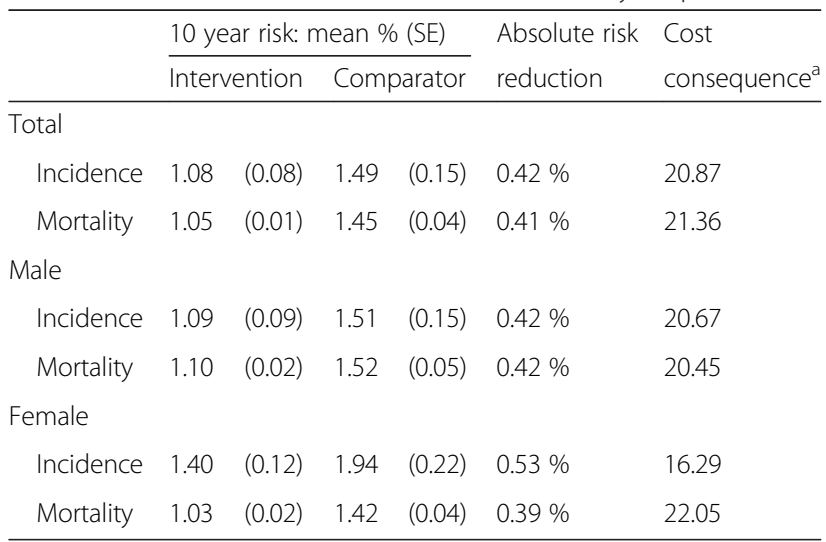

HIV human immunodeficiency virus; SE standard error

aper $1 \%$ reduction in risk identified as a strategic output of the National Service Delivery Agreement [5].

The South African epidemic is predominantly heterosexual. This work represents an over-simplification of the rather complex sexual networking structures at play in the South African HIV epidemic. Nonetheless, those individuals at high risk may still acquire infection ascribed to repeated risk exposures despite the protection conferred by the vaccine compared with those at low risk. At a population level, the premise remains that a partially effective vaccine may still avert or delay infection even if it is unable to completely prevent an infection from establishing [41]. Assessment of a partially effective vaccine in the United States of America (USA) emphasizes that even modest and temporal reductions in HIV infections have important benefits at the population level [42]. Andersson et al demonstrated similar health benefits to the USA study when modelling the RV144/Thai trial vaccine in South Africa, but cautioned that a vaccine of limited duration could only be effective with high coverage levels, which translated to millions of doses [43].

Adolescents are a critical target for this intervention. Apart from being a key population identified in the transmission of HIV, adolescents in a school environment appear more easily accessible as a target group considering that more commonly identified high risk groups such as commercial sex workers are often harder to reach due to stigma and marginalization [43]. However, adolescents have historically encountered barriers in trying to access health services in South Africa from confidentiality issues to the judgmental attitudes of staff. It is not surprising that they often do not return for follow up care [9]. The school environment could be deemed a "safe space" for peer discussion and accessibility of relevant health services. Neglecting the comprehensive health needs and barriers to 
care of this adolescent population has the potential to undermine the success of HIV prevention initiatives [44]. Further, low social acceptability of HIV vaccines fueled by the fear of vaccines and poor side effect profiles present potential deterrents to uptake and coverage [45]. It is understandably difficult for hypothetical scenarios to emulate real-life behavioral changes but knowledge of these factors underscores the need for comprehensive sexual education and risk reduction counselling; which could prove more plausible in the school environment [46].

This study had several limitations. Firstly, it is unclear to what degree behavioral disinhibition may occur following vaccination as this was not assessed in the model. Changes in sexual risk behavior post HIV vaccination are poorly understood in the African setting [46]. In high HIV prevalence communities like South Africa, a decrease in condom use even with stable partners would likely result in an increase in HIV rates [46]. In fact, South African data has inferred that poor comprehension of the 'low-efficacy' concept was associated with a reported potential decrease in condom use. It is further postulated that the degree of behavioral disinhibition may depend largely on the manner in which the vaccine effects are marketed to the public and vaccine recipients alike [40]. The impact of risk compensation becomes critical when considering the low efficacy displayed by the candidate vaccines thus far [46]. Secondly, the study was unable to assess the effects of herd immunity. Notably, Long et al. alluded to partial efficacy vaccines providing some benefits to the unvaccinated population through herd immunity [42]. This is particularly important considering the low coverage rates of childhood vaccinations in South Africa as it speaks directly to the country's capacity to introduce and implement a HIV vaccine [22]. At $60 \%$ coverage, this program calls for an unprecedented 5.9 million adolescents to be vaccinated. Given this, it is not surprising that implementation costs are high. Thirdly, the provider perspective was considered as the largest burden of direct medical program costs will be borne by the healthcare sector. Although the societal costs were not analyzed, its contribution would be substantial and could improve the overall costeffectiveness of the vaccine. Fourthly, booster vaccinations were not assessed in the original RV144/Thai trial work [42]. Therefore the assumption that booster vaccination would provide the same protective effects as the initial vaccination was hypothetical. There has been limited description of this in the literature [46]. Additionally, administration costs would drastically increase the program costs with the need for annual boosters. This is the key cost factor implicated in the difference between the comparator and intervention cost. However, it is hoped that attrition rates of vaccine recipients would be minimized by targeting the relatively stable school population. Lastly, this study has considered HIV vaccination as an isolated intervention apart from the ART rollout and condom distribution. In the clinical setting, this intervention would probably work synergistically with other prevention strategies such as male medical circumcision and an optimal combination of strategies should be better defined once data becomes available [40, 42]. As discussed earlier, the limited success achieved in curbing the national HIV incidence by the current public sector HIV prevention strategies warrants the evaluation of strategies on its individual merits.

\section{Conclusion}

In conclusion, these findings suggests that a national HIV vaccine program administered to adolescents in South Africa would be a cost-effective means for reducing the massive disease and economic burden of HIV. The implications on health outcomes are significant with reductions in HIV associated mortality and incidence and improved life expectancy demonstrated by the model. However, a vaccine with more durable protection and requiring fewer boosters would considerably reduce costs. While this work provides decision makers with objective baseline data for considering the adoption of the potential HIV vaccination intervention nationally, more realistic estimates on cost and disease burden should be gauged once the efficacy, duration of protection and vaccine cost is determined.

\section{Abbreviations}

Cl: confidence interval; GDP: Gross Domestic Product; ART: highly active antiretroviral therapy; HIV: Human Immunodeficiency Virus; ICER: incremental cost-effectiveness ratio; PHC: primary healthcare; PSA: probabilistic sensitivity analysis; QALY: quality adjusted life year; UPFS: uniform patient fee schedule; USA: United States of America; US\$: United States Dollar; ZAR: South African Rand.

\section{Competing interests}

The authors declare that they have no competing interests.

\section{Authors' contributions}

NM, MYB and GEG contributed to the conception of the study. MYB provided guidance and supported the co-ordination of the study. NM and MYB contributed to the statistical analysis. NM was responsible for the overall drafting of the manuscript. All authors contributed to critically revising its content. All authors read and approved the final manuscript.

\section{Funding}

This work was supported by the National Institute of Allergy and Infectious Diseases (NIAID) U.S. Public Health Service Grants UM1 Al068614 [LOC: HIV Vaccine Trials Network] as part of the South African HVTN AIDS Vaccine Early Stage Investigator Program (SHAPe). The support of the DST-NRF Centre of Excellence in Epidemiological Modelling and Analysis towards this research is hereby acknowledged. Opinions expressed and conclusions arrived at, are those of the author and are not necessarily to be attributed to SACEMA.

\section{Author details}

'Perinatal HIV Research Unit, Faculty of Health Sciences University of the Witwatersrand, PO Box 114 Diepkloof 1864 Johannesburg, South Africa. ${ }^{2}$ South African HVTN AIDS Vaccine Early Stage Investigator Program (SHAPe), Seattle, WA, United States. ${ }^{3}$ The South African Department of Science and 
Technology/National Research Foundation (DST/NRF) Centre of Excellence in Epidemiological Modelling and Analysis (SACEMA), University of Stellenbosch, Stellenbosch, South Africa. ${ }^{4}$ South African Medical Research Council, Tygerberg, South Africa. ${ }^{5}$ Vaccine and Infectious Disease Division, Fred Hutchinson Cancer Research Centre, Seattle, WA, USA. ${ }^{6}$ Health Systems Governance and Finance, World Health Organization, Geneva, Switzerland.

\section{Received: 10 August 2015 Accepted: 14 March 2016}

\section{Published online: 14 April 2016}

\section{References}

1. Joint United Nations Programme on HIV/AIDS. The Gap Report. In. Geneva, Switzerland: UNAIDS; 2014. http://www.unaids.org/en/resources/documents/ 2014/20140716_UNAIDS_gap_report. Accessed 10 Nov 2014.

2. Shisana O, Rehle T, Simbayi LC, Zuma K, Jooste S, Zungu N, Labadarios D, Onoya D. South African National HIV Prevalence, Incidence and Behaviour Survey, 2012. Cape Town, South Africa: HSRC Press; 2014. http://www.hsrc. ac.za/en/research-data/view/6871. Accessed 8 Dec 2014.

3. Statistics South Africa. Mid-year population estimates 2014. In. Pretoria, South Africa: Statistics South Africa; 2014 http://www.statssa.gov.za/ publications/P0302/P03022014.pdf. Accessed 9 Sep 2015.

4. South African National AIDS Council. Progress Report on the National Strategic Plan for HIV, TB and STIs (2012-2016). In. Pretoria, South Africa: SANAC; 2014. http://sanac.org.za/2015/05/25/progress-report-nationalstrategic-plan-on-hiv-stis-and-tb-2012-2016/. Accessed 19 Jul 2015.

5. National Department of Health. National Service Delivery Agreement. In. Pretoria, South Africa: National Department of Health; 2010. http:/www. thepresidency.gov.za/MediaLib/Downloads/Home/Ministries/Department ofPerformanceMonitoringandEvaluation3/TheOutcomesApproach/Health\% 20Sector\%20NSDA.pdf. Accessed 23 Mar 2015.

6. Moorhouse M. Closer to zero: relections on ten years of ART rollout. S Afr . HIV Med. 2014;15(1):9.

7. Gray A, Conradie F, Crowley T, Gaede B, Gils T, Shroufi A, Hwang B, Kegakilwe D, Nash J, Pillay P, et al. Improving access to antiretrovirals in rural South Africa - a call to action. S Afr Med J. 2015;105(8):638-9.

8. National Department of Health. National Strategic Plan on HIV, STI's and TB 2012-2016. In. Pretoria, South Africa: National Department of Health; 2011. http://www.thepresidency.gov.za/MediaLib/Downloads/Home/Publications/ SANACCallforNominations/A5summary12-12.pdf . Accessed 13 Jan 2012.

9. Nkala B, Khunwane M, Dietrich J, Otwombe K, Sekoane I, Sonqishe B, Gray G. Kganya Motsha Adolescent Centre: a model for adolescent friendly HIV management and reproductive health for adolescents in Soweto, South Africa. AIDS care. 2015;27(6):697-702.

10. Ashton J, Dickson K, Pleaner M. Evolution of the national Adolescentfriendly Clinic Initiative in South Africa. Geneva, Switzerland: WHO; 2009. http://apps.who.int/iris/bitstream/10665/44154/1/9789241598361_eng.pdf. Accessed 6 Nov 2011.

11. Lesedi C, Hoque ME, Ntuli-Ngcobo B. Youth's Perception towards Sexual and Reproductive Health Services at Family Welfare Association Centres in Botswana. J Soc Sci. 2011;28(2):137-43.

12. National Department of Health. Provincial Guidelines for the Implementation of the Three Streams of PHC Re-engineering. In. Pretoria, South Africa: National Department of Health; 2011 http://www.cmt.org.za/wp-content/uploads/2011/ 09/GUIDELINES-FOR-THE-IMPLEMENTATION-OF-THE-THREE-STREAMS-OF-PHC4-Sept-2.pdf. Accessed 6 Oct 2011.

13. Ozawa S, Mirelman A, Stack ML, Walker DG, Levine OS. Cost-effectiveness and economic benefits of vaccines in low- and middle-income countries: a systematic review. Vaccine. 2012;31(1):96-108.

14. Rerks-Ngarm S, Pitisuttithum P, Nitayaphan S, Kaewkungwal J, Paris R, Premsri N, Namwat C, de Souza M, Adams E, Benenson M, et al. Vaccination with ALVAC and AIDSVAXto Prevent HIV-1 Infection in Thailand. N Engl J Med. 2009;361(23): 2209-20.

15. Liefman LS. NIH-Sponsored HIV Vaccine Trial Launches in South Africa Early Stage Trial Aims to Build on RV144 Results. In: National Institute of Allergy and Infectious Diseases. U.S. Department of Health and Human Services; 2015. http://www.nih.gov/news-events/news-releases/nihsponsored-hiv-vaccine-trial-launches-south-africa. Accessed 16 Apr 2015)

16. Husereau D, Drummond M, Petrou S, Carswell C, Moher D, Greenberg D, Augustovski F, Briggs AH, Mauskopf J, Loder E. Consolidated Health Economic Evaluation Reporting Standards (CHEERS) statement. Cost Eff Resour Alloc. 2013;11(1):1478-7547.
17. National Department of Health. HIV Counselling and Testing (HCT) Policy Guidelines. Pretoria, South Africa: National Department of Health; 2010. http://www.genderjustice.org.za/publication/national-hiv-counselling-andtesting-hct-policy-guidelines/ . Accessed 11 Nov 2010.

18. National Department of Health. The South African Antiretroviral Treatment Guidelines 2013. Pretoria, South Africa: National Department of Health; 2013. http://www.sahivsoc.org/upload/documents/2013\%20ART\%20GuidelinesShort\%20Combined\%20FINAL\%20draft\%20guidelines\%2014\%20March\% 202013.pdf. Accessed 6 Apr 2014.

19. Tan-Torres Edejer T, Baltussen R, Adam T, Hutubessy R, Acharya A, Evans DB, Murray CLL. Making choices in health: WHO Guide to Cost Effectiveness analysis. Geneva, Switzerland: World Health Organization; 2003. http//wwww.who.int/ choice/publications/p_2003_generalised_cea.pdf. Accessed 16 Mar 2015.

20. Robberstad B. QALYS vs DALYS vs LYs gained: What are the differences, and what difference do they make for health care priority setting? Norsk Epidemiologi. 2005;15(2):183-91.

21. Russell N, Marovich M. P5 Update and GAC Progress Report. In: P5 Global Access Committee RSA Summit: October 2015; Cape Town International Conference Centre; October 2015.

22. World Health Organisation. Annual WHO/UNICEF Joint Reporting Form and WHO Regional office reports (Updates of 2013/July/13). In. Edited by Immunization Vaccines and Biologicals. Geneva, Switzerland: World Health Organization; 2013.http://www.who.int/immunization/monitoring_ surveillance/Immunization_Summary_2013.pdf. Accessed 23 Nov 2015.

23. Kardas-Nelson M, Goswami S. Upping the competition. NSP review 20136 http://www.nspreview.org/wp-content/uploads/2014/06/NSP-review-6-web. pdf. Accessed 9 Jan 2014. (May-June):26-29.

24. Nguyen A, Datta SD, Schwalbe N, Summers D, Adlide G. Working towards affordable pricing for HPV vaccines for developing countries: The role of GAVI. In: Harvard Global Equity Initiative GTFCCC Working paper and Background Series, No 3. 2011.

25. Tengs TO, Lin TH. A Meta-Analysis of Utility Estimates for HIV/AIDS. Medical decision making : an international journal of the Society for Medical Decision Making. 2002;22(6):475-81.

26. The World Bank. http://data.worldbank.org. Accessed 12 Jun 2015.

27. Badri M, Maartens G, Mandalia S, Bekker LG, Penrod JR, Platt RW, Wood R, Beck EJ. Cost-effectiveness of highly active antiretroviral therapy in South Africa. PLoS medicine. 2006;3(1), e4.

28. Sachs JD. Macroeconomics and Health: Investing in Health for Economic Development. Report of the Commission on Macroeconomics and Health. Geneva, Switzerland: World Health Organization; 2001. http://apps.who.int/ iris/bitstream/10665/42435/1/924154550X.pdf. Accessed 23 Nov 2015.

29. Salomon JA, Mathers CD, Murray CIL, Ferguson B. Methods for life expectancy and healthy life expectancy uncertainty analysis. Global Programme on Evidence for Health Policy Working Paper No. 10. Geneva: World Health Organization; 2001.

30. Mills J. Historic and Projected Mortality Data from the Period and Cohort Life Tables, 2012- based, UK, 1981-2062: Statistical Bulletin. United Kingdom: Office for National Statistics; 2013.

31. Murray CJL, Ahmad OB, Lopez AD, Salomon JA. WHO System of Model Life Tables. GPE Discussion Paper Series: No 8. Geneva, Switzerland: World Health Organization; 2000. http://www.who.int/healthinfo/paper08.pdf . Accessed 1 Nov 2015.

32. World Health Organization. WHO methods for life expectancy and healthy life expectancy. Global Health Estimates Technical Paper WHO/HIS/HSI/GHE/ 2014.5. Geneva, Switzerland: World Health Organisation; 2014. http://www. who.int/healthinfo/statistics/LT_method.pdf. Accessed 1 Nov 2015.

33. De Wet N, Oluwaseyi S, Odimegwu C. Youth mortality due to HIV/AIDS in South Africa, 2001-2009: an analysis of the levels of mortality using life table techniques. Afr J AIDS Res. 2014;13(1):13-20.

34. Dranger E, Remington P. YPLL: A Summary Measure of Premature Mortality Used in Measuring the Health of Communities. Wisconsin Public Health \& Health Policy Institute Issue Brief 2004, 5(7). https://uwphi.pophealth.wisc. edu/publications/issue-briefs/issueBriefv05n07.pdf. Accessed 23 Nov 2015.

35. Gardner JW, Sanborn JS. Years of potential life lost (YPLL)-what does it measure? Epidemiology. 1990;1(4):322-9.

36. Jain SK. Recent trends in mortality in Australia-an analysis of the causes of death through the application of life table techniques. J Aust Popul Assoc. 1992;9(1):1-23.

37. Bekker $L G$, Johnson $L$, Wallace $M$, Hosek S. Building our youth for the future. J Int AIDS Soc. 2015;18(2 Suppl 1):20027. 
38. Beksinska ME, Smit JA, Mantell JE. Progress and challenges to male and female condom use in South Africa. Sex Health. 2012;9(1):51-8.

39. Mayosi BM, Flisher AJ, Lalloo UG, Sitas F, Tollman SM, Bradshaw D. The burden of non-communicable diseases in South Africa. Lancet. 2009;374:934-47.

40. Nagelkerke NJD, Hontelez JAC, de Vlas S. The potential impact of an HIV vaccine with limited protection on HIV incidence in Thailand: A modeling study. Vaccine. 2011;29:6079-85.

41. Schneider K, Kerr CC, Hoare A, Wilson DP. Expected epidemiological impacts of introducing an HIV vaccine in Thailand: A model-based analysis. Vaccine. 2011;29:6086-91.

42. Long EF, Owens DK. The cost-effectiveness of a modestly effective HIV vaccine in the United States. Vaccine. 2011;29(36):6113-24.

43. Andersson KM, Stover J. The potential impact of a moderately effective HIV vaccine with rapidly waning protection in South Africa and Thailand. Vaccine. 2011:29(36):6092-9.

44. Sawyer SM, Afifi RA, Bearinger LH, Blakemore SJ, Dick B, Ezeh AC, Patton C. Adolescence: a foundation for future health. Lancet. 2012;379:1630-40.

45. Newman PA, Logie C. HIV vaccine acceptability: a systematic review and meta-analysis. AIDS. 2010;24(11):1749-56.

46. Andersson KM, Vardas E, Niccolai LM, Van Niekerk RM, Mogale MM, Holdsworth IM, Bogoshi M, McIntyre JA, Gray GE. Anticipated changes in sexual behaviour following vaccination with a low-efficacy HIV vaccine: survey results from a South African township. International journal of STD \& AIDS. 2012;23(10):736-41.

47. Lehtinen M, Paavonen J, Wheeler CM, Jaisamrarn U, Garland SM, Castellsague X, Skinner SR, Apter D, Naud P, Salmeron J, et al. Overall efficacy of HPV-16/18 AS04-adjuvanted vaccine against grade 3 or greater cervical intraepithelial neoplasia: 4-year end-of-study analysis of the randomised, double-blind PATRICIA trial. Lancet Oncol. 2012;13(1):89-99.

48. National Department of Health. HM08-2013SYR: The supply and delivery hypodermic syringes, needles and bloodletting devices to the Department of Health for the period 01 December 2013 to November 2015. Pretoria, South Africa: National Department of Health; 2013. http://www.health.gov. za/tender/docs/contructs/HM08-2013SYR.pdf. Accessed 23 Mar 2015.

49. National Department of Health. HM09-2014RTK: Supply and delivery of rapid test kits to the Department of Health for the period 1 April 2014 to 31 March 2017. Pretoria, South Africa: National Department of Health; 2014. http://www.health.gov.za/tender/docs/contructs/HM092014RTKCONTRACTCIRCULAR.pdf. Accessed 23 Mar 2015

50. National Department of Health. Approved UPFS 2014 Fee Schedule for Externally Funded Patients Treated at Differentiated Amenities (Private Wards at Public Health Care Facilities). Pretoria, South Africa: National Department of Health; 2014. http://www.healthinquiry.net/ Public\%20Submissions/BHF\%20AnnexureF.pdf. Accessed 23 Mar 2015.

51. National Department of Health. HM01-2012CNDM: Supply and delivery of male and female condoms to the Department of Health from 1 December 2012 to 30 November 2014. Pretoria, South Africa: National Deprtment of Health; 2012. http://www.health.gov.za/tender/docs/contructs/ HM012012CNDM02Contracts.pdf. Accessed 23 Mar 2015.

52. National Department of Health. HP03-2013FP: Supply and delivery of family planing agents to the Department of Health for the period 1 October 2013 to 30 September 2015. Pretoria, South Africa: National Department of Health; 2013. http://www.health.gov.za/tender/docs/contructs/HP03-2013FP. pdf. Accessed 23 Mar 2015

53. National Health Laboratory Services. State pricing catalogue 2013. In. Pretoria, South Africa; 2013 www.nhls.ac.za. Accessed 23 Nov 2015.

54. Johnson LF. Access to antiretroviral treatment in South Africa, 2004-2011. The Southern African Journal of HIV Medicine. 2012;13(1):22-7.

55. Fox MP, Cutsem GV, Giddy J, Maskew M, Keiser O, Prozesky H, Wood R, Hernan MA, Sterne JA, Egger M, et al. Rates and predictors of failure of firstline antiretroviral therapy and switch to second-line ART in South Africa. J Acquir Immune Defic Syndr. 2012;60(4):428-37.

56. Murphy RA, Sunpath $H$, Castilla C, Ebrahim S, Court R, Nguyen H, Kuritzkes DR, Marconi VC, Nachega JB. Second-line antiretroviral therapy: long-term outcomes in South Africa. J Acquir Immune Defic Syndr. 2012;61(2):158-63.

\section{Submit your next manuscript to BioMed Central and we will help you at every step:}

- We accept pre-submission inquiries

- Our selector tool helps you to find the most relevant journal

- We provide round the clock customer support

- Convenient online submission

- Thorough peer review

- Inclusion in PubMed and all major indexing services

- Maximum visibility for your research

Submit your manuscript at www.biomedcentral.com/submit
() BioMed Central 